\title{
A New, Young, Low-Mass Spectroscopic Binary Without a Home
}

\author{
Laura S. Flagg ${ }^{1,2}$, Evgenya L. Shkolnik ${ }^{1}$, Alycia J. Weinberger ${ }^{3}$, \\ Brendan P. Bowler ${ }^{4}$, Adam L. Kraus ${ }^{5}$, and Michael C. Liu ${ }^{6}$ \\ ${ }^{1}$ Lowell Observatory, Flagstaff, AZ 86001, USA, email: lflagg@lowell.edu \\ ${ }^{2}$ Department of Physics and Astronomy, Northern Arizona University, Flagstaff, AZ 86011, \\ USA \\ ${ }^{3}$ Department of Terrestrial Magnetism, Carnegie Institution for Science, Washington, DC \\ 20015 USA \\ ${ }^{4}$ California Institute of Technology, Pasadena, CA 91125, USA \\ ${ }^{5}$ Department of Astronomy, University of Texas at Austin, TX, USA \\ ${ }^{6}$ Institute for Astronomy, University of Hawai'i at Manoa, Honolulu, HI 96822, USA
}

\begin{abstract}
We have discovered that 2MASS 08355977-3042306 is an accreting K7, doublelined, spectroscopic binary younger than $\sim 20 \mathrm{Myr}$. The age of a dispersed young star can best be determined if it is a member of a known young moving group. However, the three dimensional space velocities $(U V W)$ we calculate using radial velocity measurements, proper motions, and plausible photometric distances make membership in any known young moving group unlikely.
\end{abstract}

Keywords. stars: low-mass, binaries: spectroscopic, stars: pre-main-sequence, stars: kinematics

The parameters of young, spectroscopic binaries are important for increasing our understanding of star formation and testing stellar evolutionary models. For stars that are 10 to $300 \mathrm{Myr}$ old, the age can be determined by classifying the system into a young moving group (YMG). As part of our broader search for 20 Myr $\beta$ Pic YMG members, we acquired two epochs of high resolution $(\mathrm{R} \approx 45,000)$ optical spectra from the $2.5 \mathrm{~m}$ Irénée du Pont telescope of 2MASS 08355977-3042306. The spectra revealed it to be a young, accreting, double-lined spectroscopic binary with a composite spectral type of K7.

The one dimensional cross-correlation function (CCF) from March 28, 2014, calculated with the IRAF task fxcor using the radial velocity (RV) standard GJ 653 (SpT=K5), showed two blended peaks in each order of the spectrum with the primary star blueshifted. The CCF has a different, asymmetric shape on January 13, 2015 where the primary peak is redshifted. By fitting two Gaussians to the March CCF (Fig. 1), we find a flux ratio of $\sim 0.7$ in the V-band. We calculate an average RV for each component of $-9 \pm 2 \mathrm{~km} / \mathrm{s}$ and $+31 \pm 3 \mathrm{~km} / \mathrm{s}$. The systemic RV, averaged over all epochs, including a recently acquired CHIRON spectrum, is $6 \pm 2 \mathrm{~km} / \mathrm{s}$.

We measure a composite spectral type of $\mathrm{K} 7+/-1$ subclass using the TiO-5 band index (Reid et al. 1995). Stars of this spectral type that have $\mathrm{H} \alpha$ in emission and lithium absorption are typically less than 20 Myr old (Chabrier \& Baraffe 1997; Barrado y Navascués \& Martín 2003). We measure a lithium equivalent width of $0.25 \AA$ and an $\mathrm{H} \alpha$ equivalent width of $-3 \AA$ (Fig. 1 ). The $\mathrm{H} \alpha$ emission has a $10 \%$ velocity width of $\sim 350 \mathrm{~km} / \mathrm{s}$, indicating that it is likely that one or both of the stars are still accreting (White \& Basri 2003). Our measurements of the K I equivalent width and CaH index both indicate low surface gravity (Shkolnik et al. 2011). This also implies that the system is young, and the stars have not yet fully contracted onto the main sequence.

The age of a dispersed young star can best be determined if it is a member of a known YMG. Using the Baraffe et. al (1998) models and an assumed age of 15 Myr, we 

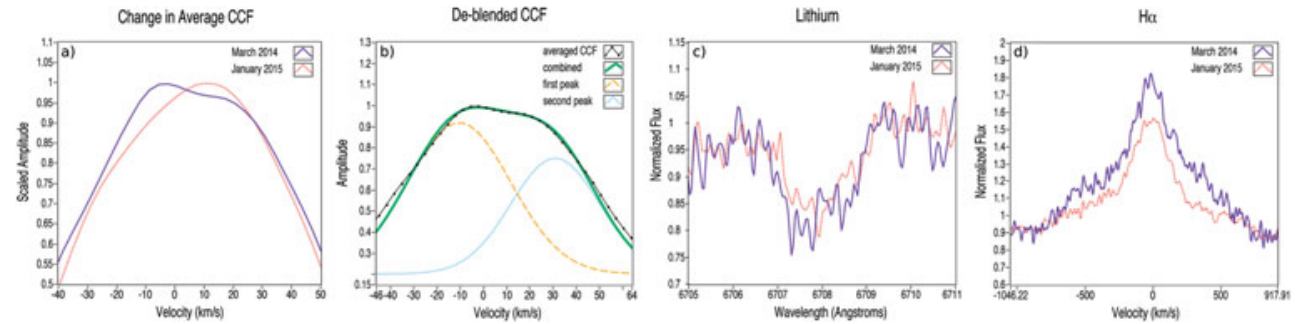

Figure 1. (a) The CCF of 2MASS $08355977-3042306$ observed on two different nights. (b) The $\mathrm{CCF}$ from March 2014 is deblended into components. (c) The equivalent width of the lithium $6707.8 \AA$ line is $0.25 \AA$. (d) The $\mathrm{H} \alpha$ emission has an equivalent width of $-3 \AA$ and the $10 \%$ velocity width is $\sim 350 \mathrm{~km} / \mathrm{s}$.
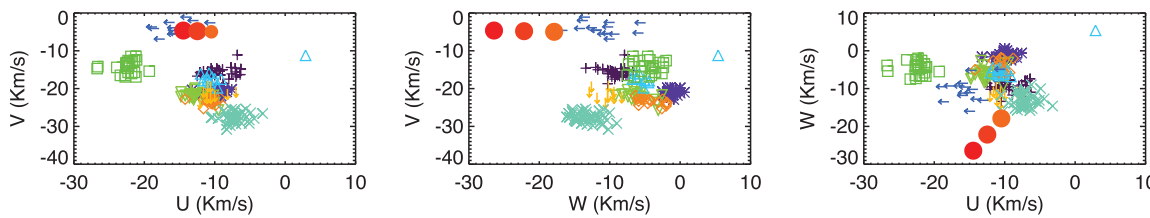

at $65 \mathrm{pc}$

eat $80 \mathrm{pc}$

at $95 \mathrm{pc}$
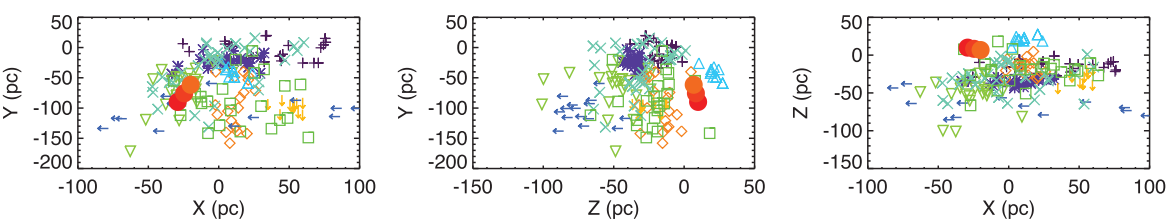

$\leftarrow$ Octans

$\times$ ABDor

Eta Cha

$\triangle$ TW Hydra

$\checkmark$ Carina

$\nabla$ Columba

*Tuc Hor

+ Beta Pic

Figure 2. The $U V W$ and $X Y Z$ values of 2MASS 08355977-3042306 for assumed photometric distances of 65,80 , and $95 \mathrm{pc}$ (colored circles), compared with the same values for various nearby YMG members. Error bars are smaller than the symbols.

calculate a photometric distance of $80 \mathrm{pc}$. There are significant uncertainties $( \pm 15 \mathrm{pc})$ associated with this distance calculated based on the $1 \sigma$ uncertainty in spectral type and the range of possible ages $(\leqslant 20 \mathrm{Myr})$. The derived proper motions are $-64.8 \mathrm{mas} / \mathrm{yr}$ in R.A. and -15.6 mas/yr in declination following the methods of Kraus and Hillenbrand (2007). Using these, we calculate the $U V W$ velocities to be $[-12.5 \pm 1.8,-4.8 \pm 2.1$, $-22.2 \pm 2.1] \mathrm{km} / \mathrm{s}$. The system's coordinates in $X Y Z$ space are $[-24.5 \pm 1.5,-75.7 \pm 5.1$, $8.3 \pm 0.5] \mathrm{pc}$. We also calculate these $U V W$ and $X Y Z$ values using distances of $95 \mathrm{pc}$ and $65 \mathrm{pc}$ to account for uncertainties. The resulting range in $U V W$ and $X Y Z$ values are indicated as circles in Fig. 2. The lack of consistent overlap with any YMG implies that membership in any of these - including $\beta$ Pic - is highly unlikely. Possible explanations for the system's apparent homelessness include that the system formed on its own, that it was kinematically ejected from a known YMG, or that it is a member of a yet to be discovered YMG.

\section{References}

Baraffe, I., Chabrier, G., Allard, F., \& Hauschildt, P. H. 1998, A\& A, 337, 403

Barrado y Navascués, D. \& Martín, E. L. 2003, AJ, 126, 2997

Chabrier, G. \& Baraffe, I. 1997, A\&A, 327, 1039

Kraus, A. L. \& Hillenbrand, L., 2007, ApJ, 134, 2340

Reid, I. N., Hawley, S. L., \& Gizis, J. E. 1995, AJ, 110, 1838

Shkolnik, E. L., Liu, M. C., Reid, I. N., Dupuy, T., \& Weinberger, A. J., 2011, ApJ, 727, 6

White, R. J. \& Basri, G. 2003, ApJ, 582, 1109 